\title{
Constructing Involutive Tableaux with Guillemin Normal Form
}

\author{
Abraham D. SMITH \\ Department of Mathematics, Statistics and Computer Science, University of Wisconsin-Stout, \\ Menomonie, WI 54751-2506, USA \\ E-mail: adsmith@member.ams.org \\ URL: http://www. curieux.us/abe/
}

Received December 15, 2014, in final form July 01, 2015; Published online July 09, 2015

http://dx.doi.org/10.3842/SIGMA.2015.053

\begin{abstract}
Involutivity is the algebraic property that guarantees solutions to an analytic and torsion-free exterior differential system or partial differential equation via the Cartan-Kähler theorem. Guillemin normal form establishes that the prolonged symbol of an involutive system admits a commutativity property on certain subspaces of the prolonged tableau. This article examines Guillemin normal form in detail, aiming at a more systematic approach to classifying involutive systems. The main result is an explicit quadratic condition for involutivity of the type suggested but not completed in Chapter IV, $\S 5$ of the book Exterior Differential Systems by Bryant, Chern, Gardner, Goldschmidt, and Griffiths. This condition enhances Guillemin normal form and characterizes involutive tableaux.
\end{abstract}

Key words: involutivity; tableau; symbol; exterior differential systems

2010 Mathematics Subject Classification: 58A15; 58H10

\section{Background}

Fix real or complex vector spaces (or bundles, etc.) $W$ and $V$ of dimension $r$ and $n$, respectively. A tableau ${ }^{1}$ is a vector space $A$ with an exact sequence

$$
0 \longrightarrow A \longrightarrow W \otimes V^{*} \stackrel{\sigma}{\longrightarrow} H^{1}(A) \longrightarrow 0
$$

A homomorphism $\sigma$ with kernel $A$ is called a symbol, taking values in the cokernel $H^{1}(A)=A^{\perp}$.

Given bases of $\left(w_{a}\right)$ of $W$ and $\left(u^{i}\right)$ of $V^{*}$, elements of $A$ are expressed as $r \times n$ matrices $\pi=\pi_{i}^{a}\left(w_{a} \otimes u^{i}\right)$. In $\left(\pi_{i}^{a}\right)$, the independent generators of $A$ appear in some $s_{1}$ entries of the first column, some $s_{2}$ entries of the second column, and so on to some $s_{n}$ entries in the last column. These numbers are constant over a Zariski-open subset of the bases (called the generic bases) of $V^{*}$, and those constant values satisfy $s_{1} \geq s_{2} \geq s_{3} \geq \cdots \geq s_{n} \geq 0$. That is, for a generic basis of $V^{*}$, the independent generators of $A$ are packed to the left in $\left(\pi_{i}^{a}\right)$. Similarly, there is a Zariski-open subset of generic bases $\left(w_{a}\right)$ of $W$ wherein the independent generators of $A$ are packed to the top. If both $\left(u^{i}\right)$ and $\left(w_{a}\right)$ are generic, then the generators of $A$ appear in the first $s_{1}$ entries of the first column, the first $s_{2}$ entries of the second column, and so on. Hence, $\operatorname{dim} A=s_{1}+s_{2}+\cdots+s_{n}$.

Given a tableau $A$, consider the tableau $A^{(1)} \rightarrow A \otimes V^{*}$ given as the kernel of the map $\delta_{\sigma}: A \otimes V^{*} \rightarrow W \otimes \wedge^{2}\left(V^{*}\right)$ by $\delta_{\sigma}=\left(1_{W} \otimes \delta\right) \circ\left(\sigma \otimes 1_{V^{*}}\right)$ where $\delta$ is the skewing map $\delta: V^{*} \otimes V^{*} \rightarrow$ $V^{*} \wedge V^{*}$. This $A^{(1)}$ is called the (first) prolonged tableau, and the map $\delta_{\sigma}$ is the (first) prolonged

\footnotetext{
${ }^{1}$ The literature contains many distinct-yet-related meanings of tableau and symbol. Usage here is consistent with [1]. In [3], Guillemin uses the phrase "a subspace of $\operatorname{Hom}(V, W)$ " where we say tableau.
} 
symbol. The cokernel is written $H^{2}(A)$,

$$
0 \longrightarrow A^{(1)} \longrightarrow A \otimes V^{*} \stackrel{\delta_{\sigma}}{\longrightarrow} W \otimes \wedge^{2} V^{*} \longrightarrow H^{2}(A) \longrightarrow 0 .
$$

It is a standard exercise to show $\operatorname{dim} A^{(1)} \leq s_{1}+2 s_{2}+\cdots+n s_{n}$. The most interesting property to study for a tableau is

Definition 1.1 (involutivity). A tableau is called involutive if and only if equality holds in the relation $\operatorname{dim} A^{(1)} \leq s_{1}+2 s_{2}+\cdots+n s_{n}$.

Verification of this equality is called Cartan's test. Its most frequent application is to check whether an overdetermined system of partial differential equations (or an exterior differential system) admits solutions to the analytic Cauchy initial-value problem. Involutivity is a coordinate-invariant property of a tableau, and techniques from homological algebra comprise most of the recent literature, for example $[2,5,6]$.

In [3], Guillemin showed involutive tableaux admit a partially commuting "normal form" for the symbol map, using Quillen's results on the exactness of Spencer cohomology from [7]. Guillemin normal form was reconsidered for exterior differential systems in [9] and [1]. This article $^{2}$ is intended to extend and clarify Guillemin normal form to aide future computational and theoretical applications regarding the geometry of partial differential equations. The main result is Theorem 3.2.

\section{Endovolutivity}

Let $\ell$ denote the index of the last non-zero Cartan character, $s_{\ell}$. Permanently reserve the index ranges $i, j, k, l \in\{1, \ldots, n\}$ and $\lambda, \mu \in\{1, \ldots, \ell\}$ and $\varrho, \varsigma \in\{\ell+1, \ldots, n\}$ and $a, b, c, d \in$ $\{1, \ldots, r\}$.

Let $\left(u^{i}\right)$ and $\left(w_{a}\right)$ denote generic bases of $V^{*}$ and $W$, respectively, so that an element $\pi \in$ $W \otimes V^{*}$ is written as a matrix $\pi=\pi_{i}^{a}\left(w_{a} \otimes u^{i}\right)$. Let $U^{*}$ denote the $\ell$-dimensional subspace spanned by $u^{1}, \ldots, u^{\ell}$. Let $Y^{*}$ denote the complementary subspace spanned by $u^{\ell+1}, \ldots, u^{n}$. We also denote the basis-dual spaces $Y=\left(U^{*}\right)^{\perp}=\left\langle u_{\ell+1}, \ldots, u_{n}\right\rangle$ and $U=\left(Y^{*}\right)^{\perp}=\left\langle u_{1}, \ldots, u_{\ell}\right\rangle$.

Using generic bases, the symbol $\sigma$ can be expressed as a minimal system of equations of the form

$$
\left\{0=\pi_{i}^{a}-B_{i, b}^{a, \lambda} \pi_{\lambda}^{b}\right\}_{s_{i}<a},
$$

where $B_{i, b}^{a, \lambda}=0$ unless $\lambda \leq i$ and $b \leq s_{\lambda}$ and $s_{i}<a$. See Fig. 1. Using the coefficients $B_{i, b}^{a, \lambda}$, we define an element of

$$
V^{*} \otimes V \otimes W \otimes W^{*} \cong \operatorname{End}\left(V^{*}\right) \otimes \operatorname{End}(W)
$$

by the tensorial expression (summed over all $\lambda, i, b$, as well as $a$ as shown)

$$
\sum_{a \leq s_{i}} \delta_{i}^{\lambda} \delta_{b}^{a}\left(w_{a} \otimes w^{b}\right) \otimes\left(u^{i} \otimes u_{\lambda}\right)+\sum_{a>s_{i}} B_{i, b}^{a, \lambda}\left(w_{a} \otimes w^{b}\right) \otimes\left(u^{i} \otimes u_{\lambda}\right) .
$$

Then, for each $\varphi=\varphi_{i} u^{i} \in V^{*}$, there is a homomorphism $B(\varphi): V \rightarrow \operatorname{End}(W)$ defined by $(2.2)$ as

$$
B(\varphi)(v)=\sum_{a \leq s_{\lambda}} \varphi_{\lambda} v^{\lambda} \delta_{b}^{a}\left(w_{a} \otimes w^{b}\right)+\sum_{a>s_{i}} \varphi_{\lambda} B_{i, b}^{a, \lambda} v^{i}\left(w_{a} \otimes w^{b}\right),
$$

\footnotetext{
${ }^{2}$ This article began as an appendix to [8], so there is some overlap in the presentation. However, be aware that some indices - such as $i, j$ - and some notations - such as $Y^{*}$ - differ between the two articles.
} 


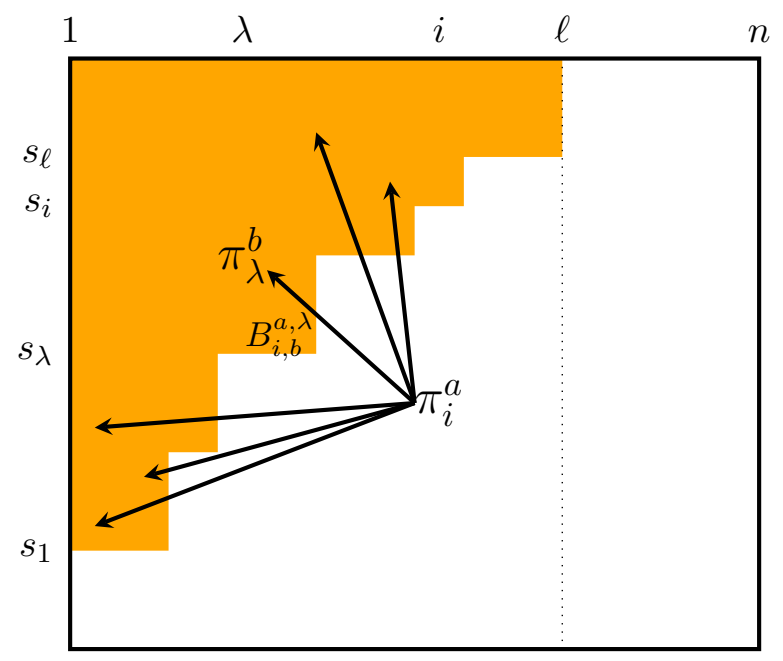

Figure 1. A tableau in coordinates, with Cartan characters $s_{1} \geq s_{2} \geq \cdots \geq s_{\ell}$. The upper-left shaded entries are independent generators. The lower-right entries depend on them via $\pi_{i}^{a}=B_{i, b}^{a, \lambda} \pi_{\lambda}^{b}$, summed as in (2.1).

where $v^{i}=u^{i}(v)$. Note that $B(\varphi)=B(\xi)$ if $\varphi_{\lambda}=\xi_{\lambda}$ for all $\lambda$, so (2.2) is really an element of $V^{*} \otimes U \otimes \operatorname{End}(W)$. We write $B_{i}^{\lambda}$ for $B\left(u^{\lambda}\right)\left(u_{i}\right)$, but note that $\left(B_{\lambda}^{i}\right)_{b}^{a}$ is not quite the same as $B_{i, b}^{a, \lambda}$ due to the identity term in (2.2); in particular,

$$
\left(B_{i}^{\lambda}\right)_{b}^{a}= \begin{cases}\delta_{b}^{a}, & \text { if } \lambda=i \text { and } a \leq s_{\lambda}, \\ B_{i, b}^{a, \lambda}, & \text { if } a>s_{i} .\end{cases}
$$

For each $i$, use the bases $\left(u^{i}\right)$ and $\left(w_{a}\right)$ to define the subspaces

$$
\mathbf{W}_{i}^{-}=\left\{z=w_{a} z^{a}: z^{a}=0 \forall a>s_{i}\right\}, \quad \mathbf{W}_{i}^{+}=\left\{z=w_{a} z^{a}: z^{a}=0 \forall a \leq s_{i}\right\} .
$$

So that $W=\mathbf{W}_{i}^{-} \oplus \mathbf{W}_{i}^{+}$and $\mathbf{W}_{1}^{-} \supset \mathbf{W}_{2}^{-} \supset \cdots \supset \mathbf{W}_{n}^{-}$according to $s_{1} \geq s_{2} \cdots \geq s_{n}$. Of course, for $\varrho>\ell$, we have $\mathbf{W}_{\varrho}^{-}=0$. For each $\lambda$, consider also the subspace

$$
\mathbf{A}_{\lambda}^{-}=\left\{\pi=B\left(u^{\lambda}\right)(\cdot) z, z \in \mathbf{W}_{\lambda}^{-}\right\} \subset A .
$$

The symbol relations (2.1) say that the coefficients $\pi_{i}^{a}$ of $\pi \in \mathbf{A}_{\lambda}^{-}$are determined by the choice of $z \in \mathbf{W}_{\lambda}^{-}$, so $\mathbf{A}_{\lambda}^{-}$and $\mathbf{W}_{\lambda}^{-}$are isomorphic via the projection onto first $s_{\lambda}$ entries in column $\lambda$. Using this basis and isomorphism, there is a decomposition

$$
A=\bigoplus_{\lambda=1}^{\ell} \mathbf{A}_{\lambda}^{-} \cong \bigoplus_{\lambda=1}^{\ell} \mathbf{W}_{\lambda}^{-} .
$$

Specifically, if $\pi=\pi_{i}^{a}\left(w_{a} \otimes u^{i}\right) \in A$, then let

$$
z_{\lambda}=\sum_{a} z_{\lambda}^{a} w_{a} \in W \quad \text { for } \quad z_{\lambda}^{a}= \begin{cases}\pi_{\lambda}^{a}, & a \leq s_{\lambda}, \\ 0, & \text { otherwise. }\end{cases}
$$

So, the decomposition (2.3) yields

$$
\pi=\sum_{\lambda} \pi_{\lambda}=\sum_{\lambda} B\left(u^{\lambda}\right)(\cdot) z_{\lambda} \in W \otimes V^{*}
$$




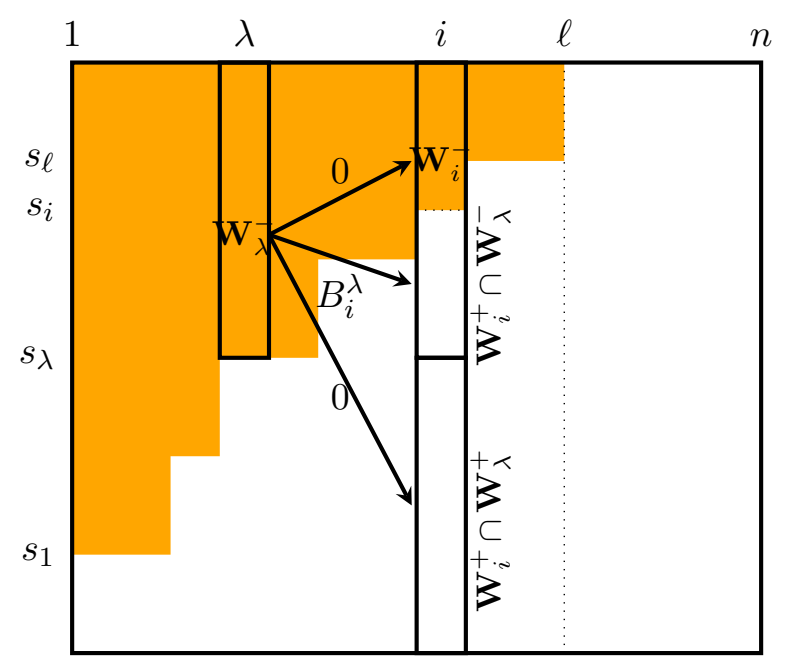

Figure 2. The map $B_{i}^{\lambda}$ for an endovolutive tableau.

Since $\operatorname{dim} \mathbf{W}_{\lambda}^{-}=s_{\lambda}$, this is a more precise version of the statement that, for a generic flag, the tableau matrix has $s_{1}$ generators in the first column, $s_{2}$ in the second column, and so on until the final $s_{\ell}$ generators in the $\ell$ column.

For any $\varphi=\varphi_{i} u^{i} \in V^{*}$, use the bases $\left(u^{i}\right)$ and $\left(w_{a}\right)$ to define the subspaces

$$
\mathbf{W}^{-}(\varphi)=\mathbf{W}_{\min \left\{i: \varphi_{i} \neq 0\right\}}^{-}, \quad \mathbf{A}^{-}(\varphi)=\left\{\pi=B(\varphi)(\cdot) z, z \in \mathbf{W}^{-}(\varphi)\right\} .
$$

Definition 2.1 (endovolutivity). A tableau expressed in generic bases of $V^{*}$ and $W$ as (2.1) is called endovolutive if and only if $B_{i, b}^{a, \lambda}=0$ for all $a>s_{\lambda}$ using those bases. See Fig. 2.

Endovolutivity is not an invariant property; a tableau could be endovolutive in generic bases $\left(u^{i}\right)$ and $\left(w_{a}\right)$ but not endovolutive in other generic bases $\left(\tilde{u}^{i}\right)$ and $\left(\tilde{w}_{a}\right)$. However, Lemma 2.2 provides that endovolutivity is invariant under the Borel subgroup of $\operatorname{GL}\left(V^{*}\right)$ that preserves the basis flag

$$
0 \subset\left\langle u^{1}\right\rangle \subset\left\langle u^{1}, u^{2}\right\rangle \subset \cdots \subset\left\langle u^{1}, \ldots, u^{n}\right\rangle=V^{*} .
$$

Lemma 2.2. Suppose a tableau $A$ is endovolutive in generic bases $\left(u^{i}\right)$ and $\left(w_{a}\right)$ for $V^{*}$ and $W$. If $\left(\tilde{u}^{i}\right)$ is another basis of $V^{*}$ related to $\left(u^{i}\right)$ through a transformation $\tilde{u}^{i}=g_{j}^{i} u^{j}$ with $g_{j}^{i}=0$ if $i>j$ (upper-triangular), then the basis $\left(\tilde{u}^{i}\right)$ is also generic for $A$, and $A$ is endovolutive in $\left(\tilde{u}^{i}\right)$ and $\left(w_{a}\right)$.

The proof is immediate by comparing Figs. 1 and 2, as the columns of $\left(\pi_{i}^{a}\right)$ are replaced with linear combinations of columns to their right.

The property of endovolutivity is discussed but not named on page 147 (page 127 in the online version) of [1] and in Section 1.2 of [9]. Endovolutivity is so-named here because of Lemma 2.3.

Lemma 2.3. A tableau expressed in generic bases is endovolutive if and only if $B(\varphi)(v)$ is an endomorphism of $\mathbf{W}^{-}(\varphi)$ for all $\varphi \in V^{*}$ and $v \in V$.

Proof. Suppose $A$ is endovolutive in the given generic bases $\left(u^{i}\right)$ and $\left(w_{a}\right)$. Fix $\varphi \in V^{*}$, and let $\kappa=\min \left\{i: \varphi_{i} \neq 0\right\}$ so that $\mathbf{W}^{-}(\varphi)=\mathbf{W}_{\kappa}^{-}$. Replacing $u^{\kappa}$ with $\varphi$ is an upper-triangular change-of-basis, so $B(\varphi)\left(u_{j}\right)=\sum_{\lambda \geq \kappa} \varphi_{\lambda} B_{j}^{\lambda}$ preserves $\mathbf{W}^{-}(\varphi)=\mathbf{W}_{\kappa}^{-}$for all $j$. Conversely, each $u^{\lambda}$ is a particular choice of $\varphi$, and $\mathbf{W}^{-}\left(u^{\lambda}\right)=\mathbf{W}_{\lambda}^{-}$, so $B\left(u^{\lambda}\right)(v)$ is an endomorphism of $\mathbf{W}^{-}\left(u^{\lambda}\right)$ if and only if $B_{i, b}^{a, \lambda}=0$ for all $a>s_{\lambda}$. 
When considering endovolutive tableaux, it useful to arrange the symbol endomorphisms as an $\ell \times n$ array of $r \times r$ matrices:

$$
\vec{B}=\left[\begin{array}{ccccccc}
I_{s_{1}} & B_{2}^{1} & B_{3}^{1} & \cdots & B_{\ell}^{1} & \cdots & B_{n}^{1} \\
0 & I_{s_{2}} & B_{3}^{2} & \cdots & B_{\ell}^{2} & \cdots & B_{n}^{2} \\
0 & 0 & I_{s_{3}} & \cdots & B_{\ell}^{3} & \cdots & B_{n}^{3} \\
& & & \ddots & & B_{i}^{\lambda} & \vdots \\
0 & 0 & 0 & 0 & I_{s_{\ell}} & \cdots & B_{n}^{\ell}
\end{array}\right] .
$$

In (2.4), each $r \times r$ matrix in row $\lambda$ is 0 outside the upper-left $s_{\lambda} \times s_{\lambda}$ part. For example, the symbol of the endovolutive tableau

$$
\pi=\left[\begin{array}{ccc}
\pi_{1}^{1} & \pi_{2}^{1} & \pi_{3}^{1} \\
\pi_{1}^{2} & \pi_{2}^{2} & Q_{4} \pi_{2}^{1}+Q_{5} \pi_{2}^{2}+T_{1} \pi_{1}^{1}+T_{2} \pi_{1}^{2}+T_{3} \pi_{1}^{3} \\
\pi_{1}^{3} & P_{1} \pi_{1}^{1}+P_{2} \pi_{1}^{2}+P_{3} \pi_{1}^{3} & R_{1} \pi_{1}^{1}+R_{2} \pi_{1}^{2}+R_{3} \pi_{1}^{3}
\end{array}\right],
$$

with $\left(s_{1}, s_{2}, s_{3}\right)=(3,2,1)$ will be arranged as

$$
\begin{aligned}
\left(\begin{array}{lll}
1 & 0 & 0 \\
0 & 1 & 0 \\
0 & 0 & 1
\end{array}\right), \quad & \left(\begin{array}{ccc}
0 & 0 & 0 \\
0 & 0 & 0 \\
P_{1} & P_{2} & P_{3}
\end{array}\right), \\
\left(\begin{array}{ccc}
1 & 0 & \\
0 & 1 &
\end{array}\right), & \left(\begin{array}{ccc}
0 & 0 & 0 \\
T_{1} & T_{2} & T_{3} \\
R_{1} & R_{2} & R_{3}
\end{array}\right), \\
& \left(\begin{array}{ccc}
0 & 0 & \\
Q_{4} & Q_{5} &
\end{array}\right), \\
& \left(\begin{array}{cc}
1 &
\end{array}\right) .
\end{aligned}
$$

Note that a change of basis in $V^{*}$ causes (2.4) to change by a block-wise conjugation. The result is not guaranteed to be endovolutive unless the change of basis is upper-triangular, like $\tilde{u}^{1}=u^{1}+\varphi_{2} u^{2}+\cdots+\varphi_{\ell} u^{\ell}$ as in the proof of Lemma 2.3:

$$
\vec{B} \mapsto\left[\begin{array}{cccc}
1 & \varphi_{2} & \cdots & \varphi_{\ell} \\
& 1 & & \\
& & \ddots & \\
& & & 1
\end{array}\right] \vec{B}\left[\begin{array}{ccccc}
1 & -\varphi_{2} & \cdots & -\varphi_{\ell} & \\
& 1 & & & \\
& & \ddots & & \\
& & & 1 & \\
& & & & I_{n-\ell}
\end{array}\right] .
$$

The goal of this article is to understand involutivity in terms of (2.4). Lemma 3.1 and Theorem 3.2 accomplish this by providing a complete version of Guillemin normal form of the type suggested but not completed in Chapter IV, $\S 5$ of [1].

\section{Involutivity}

Lemma 3.1 (linear involutivity criteria). Let $A$ denote a tableau given in a generic basis by symbol relations (2.1), as in Fig. 1. If $A$ is involutive, then $A$ admits a basis of $W$ in which it is endovolutive, as in Fig. 2.

Theorem 3.2 (quadratic involutivity criteria). Let A denote an endovolutive tableau given in a generic basis by symbol relations (2.1), as in Fig. 1. The tableau $A$ is involutive if and only if for all $b$, all $\lambda<i<j$ and $\lambda \leq \mu<j$, and all $a>s_{i}$, we have

$$
\left(B_{i}^{\lambda} B_{j}^{\mu}-B_{j}^{\lambda} B_{i}^{\mu}\right)_{b}^{a}=0 .
$$


In particular, this implies $B\left(u^{\lambda}\right)(v)$ is an endomorphism of $\mathbf{W}_{\lambda}^{-}$such that for all $v, \tilde{v} \in Y$,

$$
\left[B\left(u^{\lambda}\right)(v), B\left(u^{\lambda}\right)(\tilde{v})\right]=0 .
$$

A proof of Lemma 3.1 appears on pages 145-147 (pages 126-127 in the online version) of [1] and it is implicit in Section 1.2 of [9]. The proof of Theorem 3.2 is a lengthy inductive argument, each step of which is modeled on the approach of [9].

Proof. Choose generic bases for $W$ and $V^{*}$. As a subspace, $A \subset W \otimes V^{*}$ is defined by a minimal set of equations

$$
\pi_{i}^{a}=\sum_{b \leq s_{\lambda}} B_{i, b}^{a, \lambda} \pi_{\lambda}^{b}, \quad \forall a>s_{i}
$$

where $B_{i, b}^{a, \lambda}=0$ unless $\lambda \leq i$ and $b \leq s_{\lambda}$. Moreover, we assume that the basis of $W$ is endovolutive, so that $B_{i, b}^{a, \lambda}=0$ if $a>s_{\lambda}$.

Let $\left\{z_{i}^{a}, a \leq s_{i}\right\}$ be a basis for the abstract vector space $A^{*}$. Define a monomorphism $A \rightarrow W \otimes V^{*}$ by

$$
\begin{aligned}
& \pi_{1}^{a}=B_{1, b}^{a, 1} z_{1}^{b}, \\
& \pi_{2}^{a}=B_{2, b}^{a, 1} z_{1}^{b}+B_{2, b}^{a, 2} z_{2}^{b}, \\
& \pi_{3}^{a}=B_{3, b}^{a, 1} z_{1}^{b}+B_{3, b}^{a, 2} z_{2}^{b}+B_{3, b}^{a, 3} z_{3}^{b}, \\
& \vdots \\
& \pi_{n}^{a}=B_{n, b}^{a, 1} z_{1}^{b}+B_{n, b}^{a, 2} z_{2}^{b}+\cdots \quad \cdots+B_{n, b}^{a, n} z_{n}^{b} .
\end{aligned}
$$

We set $B_{\lambda, b}^{a, \lambda}=\delta_{b}^{a}$ for $a \leq s_{\lambda}$, so that (3.1) satisfies (2.1).

The prolongation $A^{(1)} \subset A \otimes V^{*}$ is given by coefficients $\left\{Z_{i, j}^{a}, a \leq s_{i}\right\}$ with the "contact" system taking the form $z_{i}^{a}=Z_{i, j}^{a} u^{j}$. By Cartan's test, the condition of involutivity means that exactly $s_{1}+2 s_{2}+\cdots+n s_{n}$ of these coefficients are independent functions on $A^{(1)}$.

This proof is based on Section 1.1 of [9], where it is shown that a tableau is involutive precisely when, in generic bases, the usual 2-form condition

$$
0 \equiv \pi_{1}^{a} \wedge u^{1}+\pi_{2}^{a} \wedge u^{2}+\cdots+\pi_{n}^{a} \wedge u^{n}
$$

is equivalent to the sequence of conditions

$$
\begin{aligned}
& 0 \equiv \pi_{1}^{a} \wedge u^{1} \quad \bmod u^{2}, \ldots, u^{n}, \\
& 0 \equiv \pi_{1}^{a} \wedge u^{1}+\pi_{2}^{a} \wedge u^{2} \quad \bmod u^{3}, \ldots, u^{n}, \\
& \vdots \quad \ddots \\
& 0 \equiv \pi_{1}^{a} \wedge u^{1}+\pi_{2}^{a} \wedge u^{2}+\cdots+\pi_{k}^{a} \wedge u^{k} \quad \bmod u^{k+1}, \ldots, u^{n} \\
& 0 \equiv \pi_{1}^{a} \wedge u^{1}+\pi_{2}^{a} \wedge u^{2}+\cdots \quad \cdots+\pi_{n}^{a} \wedge u^{n} .
\end{aligned}
$$

The following argument shows that this sequence of conditions forces $Z_{i, j}^{a}$ with $a \leq s_{i}$ and $j \leq i$ to be a complete set of independent generators of $A^{(1)}$, providing it with a dimension of $s_{1}+2 s_{2}+3 s_{3}+\cdots+n s_{n}$. To emphasize these terms in the following computations, we underline them. 
The proof proceeds by induction, sequentially verifying that each term of each row of (3.2) yields a condition of the desired form. One may interpret this as induction over $n$ of Cartan's test for all tableaux of size $r \times n$.

Row 1 of (3.2) prolongs to

$$
0=\pi_{1}^{a} \wedge u^{1}=B_{1, b}^{a, 1} z_{1}^{b} \wedge u^{1}=B_{1, b}^{a, 1} Z_{1, i}^{b} u^{i} \wedge u^{1} \equiv B_{1, b}^{a, 1} \underline{Z_{1,1}^{b}} u^{1} \wedge u^{1} \quad \bmod u^{2}, \ldots, u^{n},
$$

which is trivial. Every tableau with $n=1$ is involutive. The $Z_{1,1}^{b}$ terms account for $s_{1}$ generators of $A^{(1)}$.

Row 2 of (3.2) prolongs to

$$
\begin{aligned}
0 & =\pi_{1}^{a} \wedge u^{1}+\pi_{2}^{a} \wedge u^{2}=\left(B_{1, b}^{a, 1} z_{1}^{b}\right) \wedge u^{1}+\left(B_{2, b}^{a, 1} z_{1}^{b}+B_{2, b}^{a, 2} z_{2}^{b}\right) \wedge u^{2} \\
& =\left(B_{1, b}^{a, 1} Z_{1, i}^{b}\right) u^{i} \wedge u^{1}+\left(B_{2, b}^{a, 1} Z_{1, i}^{b}+B_{2, b}^{a, 2} Z_{2, i}^{b}\right) u^{i} \wedge u^{2} \\
& \equiv\left(B_{2, b}^{a, 1} \underline{Z_{1,1}^{b}}+B_{2, b}^{a, 2} \underline{Z_{2,1}^{b}}-B_{1, b}^{a, 1} Z_{1,2}^{b}\right) u^{1} \wedge u^{2} \quad \bmod u^{3}, \ldots, u^{n} .
\end{aligned}
$$

Endovolutivity implies this is trivial when projected to $\mathbf{W}_{1}^{+}$(meaning "for $a>s_{1}$ "). But, when considering the projection to $\mathbf{W}_{1}^{-}$, we see the condition

$$
Z_{1,2}^{a}=B_{2, b}^{a, 1} \underline{Z_{1,1}^{b}}+B_{2, b}^{a, 2} \underline{Z_{2,1}^{b}}, \quad \forall a \leq s_{1} .
$$

The $Z_{2,1}^{b}$ and $Z_{2,2}^{b}$ terms account for $2 s_{2}$ new generators of $A^{(1)}$. So far, there is no quadratic

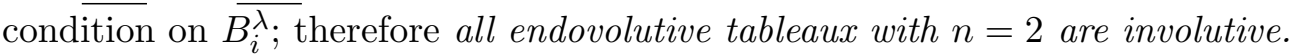

Notation! It is clear we must confront a proliferation of indices $a, b, c, \ldots$ covering $W$. Henceforth, we suppress these indices and work directly on $W$-valued objects. Instead of saying " $\forall a \leq s_{i}$ " and " $\forall a>s_{i}$," we say "on $\mathbf{W}_{i}^{-}$" and "on $\mathbf{W}_{i}^{+}$," respectively. Note that this always refers to projection on the range of the expression, not a restriction of its domain; by our definition of $B_{i}^{\lambda}$, we may assume the domain is always $W$.

Row 3 of (3.2) prolongs to

$$
\begin{aligned}
0= & \pi_{1} \wedge u^{1}+\pi_{2} \wedge u^{2}+\pi_{3} \wedge u^{3} \\
= & \left(B_{1}^{1} z_{1}\right) \wedge u^{1}+\left(B_{2}^{1} z_{1}+B_{2}^{2} z_{2}\right) \wedge u^{2}+\left(B_{3}^{1} z_{1}+B_{3}^{2} z_{2}+B_{3}^{3} z_{3}\right) \wedge u^{3} \\
= & \left(B_{1}^{1} Z_{1, i}\right) u^{i} \wedge u^{1}+\left(B_{2}^{1} Z_{1, i}+B_{2}^{2} Z_{2, i}\right) u^{i} \wedge u^{2}+\left(B_{3}^{1} Z_{1, i}+B_{3}^{2} Z_{2, i}+B_{3}^{3} Z_{3, i}\right) u^{i} \wedge u^{3} \\
\equiv & \left(B_{2}^{1} Z_{1,1}+B_{2}^{2} Z_{2,1}-B_{1}^{1} Z_{1,2}\right) u^{1} \wedge u^{2}+\left(B_{3}^{1} \underline{Z_{1,1}}+B_{3}^{2} \underline{Z_{2,1}}+B_{3}^{3} \underline{Z_{3,1}}-B_{1}^{1} Z_{1,3}\right) u^{1} \wedge u^{3} \\
& +\left(B_{3}^{2} \underline{Z_{2,2}}+B_{3}^{1} Z_{1,2}+B_{3}^{3} \underline{Z_{3,2}}-B_{2}^{1} Z_{1,3}-B_{2}^{2} Z_{2,3}\right) u^{2} \wedge u^{3} \quad \underline{\bmod } u^{4}, \ldots, u^{n} .
\end{aligned}
$$

For this to vanish each component $u^{i} \wedge u^{j}$ with $i<j$ must vanish separately. The $u^{1} \wedge u^{2}$ term repeats conditions already seen in row 2 , namely

$$
Z_{1,2}=B_{2}^{i} \underline{Z_{i, 1}} \quad \text { on } \mathbf{W}_{1}^{-} \text {. }
$$

The $u^{1} \wedge u^{3}$ term is similar,

$$
Z_{1,3}=B_{3}^{i} \underline{Z_{i, 1}} \quad \text { on } \mathbf{W}_{1}^{-} \text {. }
$$

The $u^{2} \wedge u^{3}$ term is more interesting, because it requires expansion using the previous relations:

$$
\begin{aligned}
Z_{2,3} & =B_{3}^{1} Z_{1,2}+B_{3}^{2} \underline{Z_{2,2}}+B_{3}^{3} \underline{Z_{3,2}}-B_{2}^{1} Z_{1,3} \\
& =B_{3}^{1}\left(B_{2}^{1} \underline{Z_{1,1}}+B_{2}^{2} \underline{Z_{2,1}}\right)+B_{3}^{2} \underline{Z_{2,2}}+B_{3}^{3} \underline{Z_{3,2}}-B_{2}^{1}\left(B_{3}^{1} \underline{Z_{1,1}}+B_{3}^{2} \underline{Z_{2,1}}+B_{3}^{3} \underline{Z_{3,1}}\right)
\end{aligned}
$$




$$
\begin{aligned}
= & \left(B_{3}^{1} B_{2}^{1}-B_{2}^{1} B_{3}^{1}\right) \underline{Z_{1,1}}+\left(B_{3}^{1} B_{2}^{2}-B_{2}^{1} B_{3}^{2}\right) \underline{Z_{2,1}} \\
& +\left(B_{3}^{1} B_{2}^{3}-B_{2}^{1} B_{3}^{3}\right) \underline{Z_{3,1}}+B_{3}^{2} \underline{Z_{2,2}}+B_{3}^{3} \underline{Z_{3,2}} \text { on } \mathbf{W}_{1}^{-} .
\end{aligned}
$$

On $\mathbf{W}_{2}^{-}$equation (3.3) merely shows how $Z_{2,3}$ depends on the previous coordinates on $A^{(1)}$. Note that the $Z_{3,1}, Z_{3,2}$ and $Z_{3,3}$ terms contribute another $3 s_{3}$ generators of $A^{(1)}$. Cartan's test fails if and only if other relations appear among the generators $Z_{i, j}, i \geq j$. However, on $\mathbf{W}_{2}^{+}$, many of the terms vanish by definition, and the rest impose a new quadratic condition:

$$
0=\left(B_{3}^{1} B_{2}^{1}-B_{2}^{1} B_{3}^{1}\right) \underline{Z_{1,1}}+\left(B_{3}^{1} B_{2}^{2}-B_{2}^{1} B_{3}^{2}\right) \underline{Z_{2,1}}+\left(B_{3}^{1} B_{2}^{3}-B_{2}^{1} B_{3}^{3}\right) \underline{Z_{3,1}} \quad \text { on } \mathbf{W}_{2}^{+} .
$$

Therefore an endovolutive tableau with $n=3$ is involutive if and only if each term of (3.4) holds on $\mathbf{W}_{2}^{+}$.

It is useful to see another case, where things become more interesting.

Row 4 of (3.2) prolongs to

$$
\begin{aligned}
0= & \pi_{1} \wedge u^{1}+\pi_{2} \wedge u^{2}+\pi_{3} \wedge u^{3}+\pi_{4} \wedge u^{4} \\
= & \left(B_{1}^{1} z_{1}\right) \wedge u^{1}+\left(B_{2}^{1} z_{1}+B_{2}^{2} z_{2}\right) \wedge u^{2}+\left(B_{3}^{1} z_{1}+B_{3}^{2} z_{2}+B_{3}^{3} z_{3}\right) \wedge u^{3} \\
& +\left(B_{4}^{1} z_{1}+B_{4}^{2} z_{2}+B_{4}^{3} z_{3}+B_{4}^{4} z_{4}\right) \wedge u^{4} \\
= & \left(B_{1}^{1} Z_{1, i}\right) u^{i} \wedge u^{1}+\left(B_{2}^{1} Z_{1, i}+B_{2}^{2} Z_{2, i}\right) u^{i} \wedge u^{2}+\left(B_{3}^{1} Z_{1, i}+B_{3}^{2} Z_{2, i}+B_{3}^{3} Z_{3, i}\right) u^{i} \wedge u^{3} \\
& +\left(B_{4}^{1} Z_{1, i}+B_{4}^{2} Z_{2, i}+B_{4}^{3} Z_{3, i}+B_{4}^{4} Z_{4, i}\right) u^{i} \wedge u^{4} .
\end{aligned}
$$

After expanding these terms, modulo $u^{5}, \ldots, u^{n}$, the several conditions are found. From the $u^{1} \wedge u^{4}$ term:

$$
Z_{1,4}=B_{4}^{1} \underline{Z_{1,1}}+B_{4}^{2} \underline{Z_{2,1}}+B_{4}^{3} \underline{Z_{3,1}}+B_{4}^{4} \underline{Z_{4,1}} .
$$

This term imposes no quadratic conditions.

From the $u^{2} \wedge u^{4}$ term:

$$
\begin{aligned}
Z_{2,4}= & B_{4}^{1} Z_{1,2}+B_{4}^{2} \underline{Z_{2,2}}+B_{4}^{3} \underline{Z 3,2}_{3}+B_{4}^{4} \underline{Z_{4,2}}-B_{2}^{1} Z_{1,4} \\
= & B_{4}^{1}\left(B_{2}^{1} \underline{Z_{1,1}}+B_{2}^{2} \underline{Z_{2,1}}\right)+B_{4}^{2} \underline{Z_{2,2}}+B_{4}^{3} \underline{Z_{3,2}}+B_{4}^{4} \underline{Z_{4,2}} \\
& -B_{2}^{1}\left(B_{4}^{1} \underline{Z_{1,1}}+B_{4}^{2} \underline{Z_{2,1}}+B_{4}^{3} \underline{Z_{3,1}}+B_{4}^{4} \underline{Z_{4,1}}\right) .
\end{aligned}
$$

Equation (3.5) becomes a new quadratic condition when projected on $\mathbf{W}_{2}^{+}$:

$$
\begin{aligned}
0= & B_{4}^{1}\left(B_{2}^{1} \underline{Z_{1,1}}+B_{2}^{2} \underline{Z_{2,1}}\right)-B_{2}^{1}\left(B_{4}^{1} \underline{Z_{1,1}}+B_{4}^{2} \underline{Z_{2,1}}+B_{4}^{3} \underline{Z_{3,1}}+B_{4}^{4} \underline{Z_{4,1}}\right) \\
= & \left(B_{4}^{1} B_{2}^{1}-B_{2}^{1} B_{4}^{1}\right) \underline{Z_{1,1}}+\left(B_{4}^{1} B_{2}^{2}-B_{2}^{1} B_{4}^{2}\right) \underline{Z_{2,1}}+\left(B_{4}^{1} \overline{B_{2}^{3}-} B_{2}^{1} B_{4}^{3}\right) \underline{Z_{3,1}} \\
& +\left(B_{4}^{1} B_{2}^{4}-B_{2}^{1} B_{4}^{4}\right) \underline{Z_{4,1}} \quad \text { on } \mathbf{W}_{2}^{+} .
\end{aligned}
$$

When reading (3.6), recall that $B_{i}^{\lambda}=0$ if $i<\lambda$.

The $u^{3} \wedge u^{4}$ term becomes

$$
\begin{aligned}
Z_{3,4}= & B_{4}^{1} Z_{1,3}+B_{4}^{2} Z_{2,3}+B_{4}^{3} \underline{Z_{3,3}}+B_{4}^{4} Z_{4,3}-B_{3}^{1} Z_{1,4}-B_{3}^{2} Z_{2,4} \\
= & B_{4}^{1}\left(B_{3}^{1} \underline{Z_{1,1}}+B_{3}^{2} \underline{Z_{2,1}}+B_{3}^{3} Z_{3,1}\right)+B_{4}^{2}\left(B_{3}^{1}\left(B_{2}^{1} \underline{Z_{1,1}}+B_{2}^{2} \underline{Z_{2,1}}\right)+B_{3}^{2} \underline{Z_{2,2}}+B_{3}^{3} \underline{Z_{3,2}}\right. \\
& \left.-B_{2}^{1}\left(B_{3}^{1} \underline{Z_{1,1}}+B_{3}^{2} \underline{Z_{2,1}}+B_{3}^{3} \underline{Z_{3,1}}\right)\right)+B_{4}^{3} \underline{Z_{3,3}}+B_{4}^{4} \underline{Z_{4,3}} \\
& -B_{3}^{1}\left(B_{4}^{1} \underline{Z_{1,1}}+B_{4}^{2} \underline{Z_{2,1}}+B_{4}^{3} \underline{Z_{3,1}}+B_{4}^{4} \underline{Z_{4,1}}\right)-B_{3}^{2}\left(B_{4}^{1}\left(B_{2}^{1} \underline{Z_{1,1}}+B_{2}^{2} \underline{Z_{2,1}}\right)+B_{4}^{2} \underline{Z_{2,2}}\right.
\end{aligned}
$$




$$
\left.+B_{4}^{3} \underline{Z_{3,2}}+B_{4}^{4} \underline{Z_{4,2}}-B_{2}^{1}\left(B_{4}^{1} \underline{Z_{1,1}}+B_{4}^{2} \underline{Z_{2,1}}+B_{4}^{3} \underline{Z_{3,1}}+B_{4}^{4} \underline{Z_{4,1}}\right)\right) .
$$

Equation (3.7) becomes a new quadratic condition when projected on $\mathbf{W}_{3}^{+}$:

$$
\begin{aligned}
0= & B_{4}^{1}\left(B_{3}^{1} \underline{Z_{1,1}}+B_{3}^{2} \underline{Z_{2,1}}+B_{3}^{3} \underline{Z_{3,1}}\right)+B_{4}^{2}\left(B_{3}^{1}\left(B_{2}^{1} \underline{Z_{1,1}}+B_{2}^{2} \underline{Z_{2,1}}\right)+B_{3}^{2} \underline{Z_{2,2}}+B_{3}^{3} \underline{Z_{3,2}}\right. \\
& \left.-B_{2}^{1}\left(B_{3}^{1} Z_{1,1}+B_{3}^{2} Z_{2,1}+B_{3}^{3} Z_{3,1}\right)\right)-B_{3}^{1}\left(B_{4}^{1} \underline{Z_{1,1}}+B_{4}^{2} \underline{Z_{2,1}}+B_{4}^{3} \underline{Z_{3,1}}+B_{4}^{4} \underline{Z_{4,1}}\right) \\
& -B_{3}^{2}\left(B_{4}^{1}\left(B_{2}^{1} \underline{Z_{1,1}}+B_{2}^{2} \underline{Z_{2,1}}\right)+B_{4}^{2} \underline{Z_{2,2}}+B_{4}^{3} \underline{Z_{3,2}}+B_{4}^{4} \underline{Z_{4,2}}\right. \\
& \left.-B_{2}^{1}\left(B_{4}^{1} \underline{Z_{1,1}}+B_{4}^{2} \underline{Z_{2,1}}+B_{4}^{3} \underline{Z_{3,1}}+B_{4}^{4} \underline{Z_{4,1}}\right)\right) \text { on } \underline{\mathbf{W}_{3}^{+} .}
\end{aligned}
$$

This looks like a mess, but collecting terms reveals a pattern:

$$
\begin{aligned}
0= & \left(B_{4}^{1} B_{3}^{1}-B_{3}^{1} B_{4}^{1}+B_{4}^{2}\left(B_{3}^{1} B_{2}^{1}-B_{2}^{1} B_{3}^{1}\right)-B_{3}^{2}\left(B_{4}^{1} B_{2}^{1}-B_{2}^{1} B_{4}^{1}\right)\right) \underline{Z_{1,1}} \\
& +\left(B_{4}^{1} B_{3}^{2}-B_{3}^{1} B_{4}^{2}+B_{4}^{2}\left(B_{3}^{1} B_{2}^{2}-B_{2}^{1} B_{3}^{2}\right)-B_{3}^{2}\left(B_{4}^{1} B_{2}^{2}-B_{2}^{1} B_{4}^{2}\right)\right) \underline{Z_{2,1}} \\
& +\left(B_{4}^{2} B_{3}^{2}-B_{3}^{2} B_{4}^{2}\right) \underline{Z_{2,2}}+\left(B_{4}^{1} B_{3}^{3}-B_{3}^{1} B_{4}^{3}+B_{4}^{2}\left(B_{3}^{1} B_{2}^{3}-B_{2}^{1} B_{3}^{3}\right)\right. \\
& \left.+B_{3}^{2}\left(B_{4}^{1} B_{2}^{3}-B_{2}^{1} B_{4}^{3}\right)\right) \underline{Z_{3,1}}+\left(B_{4}^{2} B_{3}^{3}-B_{3}^{2} B_{4}^{3}\right) \underline{Z_{3,2}} \\
& +\left(B_{4}^{1} B_{3}^{4}-B_{3}^{1} B_{4}^{4}+B_{3}^{2}\left(B_{4}^{1} B_{2}^{4}-B_{2}^{1} B_{4}^{4}\right)\right) \underline{Z_{4,1}}+\left(B_{4}^{2} B_{3}^{4}-B_{3}^{2} B_{4}^{4}\right) \underline{Z_{4,2}} \text { on } \mathbf{W}_{3}^{+} .
\end{aligned}
$$

Notice that, using the quadratic relations already discovered for $n=2$ and $n=3$, we obtain

$$
\begin{aligned}
0= & \left(B_{4}^{1} B_{3}^{1}-B_{3}^{1} B_{4}^{1}\right) \underline{Z_{1,1}}+\left(B_{4}^{1} B_{3}^{2}-B_{3}^{1} B_{4}^{2}\right) \underline{Z_{2,1}}+\left(B_{4}^{2} B_{3}^{2}-B_{3}^{2} B_{4}^{2}\right) \underline{Z_{2,2}} \\
& +\left(B_{4}^{1} B_{3}^{3}-B_{3}^{1} B_{4}^{3}\right) \underline{Z_{3,1}}+\left(B_{4}^{2} B_{3}^{3}-B_{3}^{2} B_{4}^{3}\right) \underline{Z_{3,2}}+\left(B_{4}^{1} B_{3}^{4}-B_{3}^{1} B_{4}^{4}\right) \underline{Z_{4,1}} \\
& +\left(B_{4}^{2} B_{3}^{4}-B_{3}^{2} B_{4}^{4}\right) \underline{Z_{4,2}} \quad \text { on } \mathbf{W}_{3}^{+} .
\end{aligned}
$$

The generators $Z_{4,1}, Z_{4,2}, Z_{4,3}$, and $Z_{4,4}$ provide $A^{(1)}$ with another $4 s_{4}$ dimensions. Cartan's test fails if and only if other relations appear among the generators $Z_{i, j}, i \geq j$. An endovolutive tableau with $n=4$ is involutive if and only if each term of (3.4) and (3.6) holds on $\mathbf{W}_{2}^{+}$and each term of (3.8) holds on $\mathbf{W}_{3}^{+}$.

Inductive hypothesis. Fix $k$ and $l<k$. Assume for induction that the following are equivalent

(i) The first $k-1$ rows of the 2 -form condition (3.2) are satisfied.

(ii) The $s_{1}+2 s_{2}+\cdots+(k-1) s_{k-1}$ elements $Z_{j, i}^{a}$ of $A^{(1)}$ with $a \leq s_{j}$ and $i \leq j<k$ are independent.

More precisely, examining each row of (3.2) in detail, assume for induction that the following are equivalent

(i) The first $k-1$ rows of the 2 -form condition (3.2) are satisfied, and the $u^{i} \wedge u^{k}$ terms vanish in the $k$ th row for all $i<l$.

(ii) For all $(j, i)<(k, l)$ in lexicographic ordering on pairs $\{(j, i), i \leq j\}$, we have

$$
Z_{i, j}-\sum_{\mu=i}^{j} B_{j}^{\mu} \underline{Z_{\mu, i}}=\sum_{\lambda=1}^{i-1}\left(B_{j}^{\lambda} Z_{\lambda, i}-B_{i}^{\lambda} Z_{\lambda, j}\right) \quad \text { on } \mathbf{W}_{i}^{-},
$$

and for all $\lambda<i<j$ and $\lambda \leq \mu \leq j$, we have

$$
B_{i}^{\lambda} B_{j}^{\mu}-B_{j}^{\lambda} B_{i}^{\mu}=0 \quad \text { on } \mathbf{W}_{i}^{+} .
$$


To perform the inductive step, we compute the $u^{l} \wedge u^{k}$ terms in the $k$ th row of (3.2):

$$
\begin{aligned}
0 & =\pi_{1} \wedge u^{1}+\cdots+\pi_{l} \wedge u^{l}+\cdots+\pi_{k} \wedge u^{k} \\
& =\cdots+\left(\sum_{\lambda \leq l} B_{l}^{\lambda} z_{\lambda}\right) \wedge u^{l}+\cdots+\left(\sum_{\mu \leq k} B_{k}^{\mu} z_{\mu}\right) \wedge u^{k} \\
& \equiv\left(\sum_{\lambda \leq l} B_{l}^{\lambda} Z_{\lambda, k}\right) u^{k} \wedge u^{l}+\left(\sum_{\mu \leq k} B_{k}^{\mu} Z_{\mu, l}\right) u^{l} \wedge u^{k} \bmod u^{k+1}, \ldots, u^{n} .
\end{aligned}
$$

What follows is a tedious expansion and reduction of (3.11) using (3.9) and (3.10), along the lines of what was performed for (3.3), (3.7) and (3.5) above. The goal is to expand (3.11) in terms of the elements $Z_{j, i}$ for $j \geq i$ that remain independent if and only if Cartan's test holds.

For a little bit of sanity in the expansion that follows, we break up these sums using the index ranges $\lambda_{0}=1, \ldots, l-1$, and $\mu_{0}=l, \ldots, k$. Moreover, for every $p \geq 0$, we have nested index ranges $\lambda_{p+1}=1, \ldots, \lambda_{p}-1$ and $\mu_{p+1}=\lambda_{p}, \ldots, k$.

The vanishing of the $u^{l} \wedge u^{k}$ term of the $k$ th row of (3.2) is equivalent to

$$
0=B_{k}^{\lambda_{0}} Z_{\lambda_{0}, l}+B_{k}^{\mu_{0}} \underline{Z_{\mu_{0}, l}}-B_{l}^{\lambda_{0}} Z_{\lambda_{0}, k}-B_{l}^{l} Z_{l, k} .
$$

Rearranging terms,

$$
Z_{l, k}-B_{k}^{\mu_{0}} \underline{Z_{\mu_{0}, l}}=B_{k}^{\lambda_{0}} Z_{\lambda_{0}, l}-B_{l}^{\lambda_{0}} Z_{\lambda_{0}, k}
$$

and expanding the right-hand side by the inductive hypothesis,

$$
=B_{k}^{\lambda_{0}}\left(B_{l}^{\lambda_{1}} Z_{\lambda_{1}, \lambda_{0}}-B_{\lambda_{0}}^{\lambda_{1}} Z_{\lambda_{1}, l}+B_{l}^{\mu_{1}} \underline{Z_{\mu_{1}, \lambda_{0}}}\right)-B_{l}^{\lambda_{0}}\left(B_{k}^{\lambda_{1}} Z_{\lambda_{1}, \lambda_{0}}-B_{\lambda_{0}}^{\lambda_{1}} Z_{\lambda_{1}, k}+B_{k}^{\mu_{1}} \underline{Z_{\mu_{1}, \lambda_{0}}}\right)
$$

and expanding again by inductive hypothesis,

$$
\begin{aligned}
= & \left(B_{k}^{\lambda_{0}} B_{l}^{\mu_{1}}-B_{l}^{\lambda_{0}} B_{k}^{\mu_{1}}\right) \underline{Z_{\mu_{1}, \lambda_{0}}}+B_{k}^{\lambda_{0}}\left(B_{l}^{\lambda_{1}}\left(B_{\lambda_{0}}^{\lambda_{2}} Z_{\lambda_{2}, \lambda_{1}}-B_{\lambda_{1}}^{\lambda_{2}} Z_{\lambda_{2}, \lambda_{0}}+B_{\lambda_{0}}^{\mu_{2}} \underline{Z_{\mu_{2}, \lambda_{1}}}\right)\right. \\
& \left.-B_{\lambda_{0}}^{\lambda_{1}}\left(B_{l}^{\lambda_{2}} Z_{\lambda_{2}, \lambda_{1}}-B_{\lambda_{1}}^{\lambda_{2}} Z_{\lambda_{2}, l}+B_{l}^{\mu_{2}} \underline{Z_{\mu_{2}, \lambda_{1}}}\right)\right) \\
& -B_{l}^{\lambda_{0}}\left(B_{k}^{\lambda_{1}}\left(B_{\lambda_{0}}^{\lambda_{2}} Z_{\lambda_{2}, \lambda_{1}}-B_{\lambda_{1}}^{\lambda_{2}} Z_{\lambda_{2}, \lambda_{0}}+B_{\lambda_{0}}^{\mu_{2}} \underline{Z_{\mu_{2}, \lambda_{1}}}\right)\right. \\
& \left.-B_{\lambda_{0}}^{\lambda_{1}}\left(B_{k}^{\lambda_{2}} Z_{\lambda_{2}, \lambda_{1}}-B_{\lambda_{1}}^{\lambda_{2}} Z_{\lambda_{2}, k}+B_{k}^{\mu_{2}} \underline{Z_{\mu_{2}, \lambda_{1}}}\right)\right)
\end{aligned}
$$

and rearranging,

$$
\begin{aligned}
= & \left(B_{k}^{\lambda_{0}} B_{l}^{\mu_{1}}-B_{l}^{\lambda_{0}} B_{k}^{\mu_{1}}\right) \underline{Z_{\mu_{1}, \lambda_{0}}} \\
& +\left[B_{k}^{\lambda_{0}}\left(B_{l}^{\lambda_{1}} B_{\lambda_{0}}^{\lambda_{2}}-B_{\lambda_{0}}^{\lambda_{1}} B_{l}^{\lambda_{2}}\right)-B_{l}^{\lambda_{0}}\left(B_{k}^{\lambda_{1}} B_{\lambda_{0}}^{\lambda_{2}}-B_{\lambda_{0}}^{\lambda_{1}} B_{k}^{\lambda_{2}}\right)\right] Z_{\lambda_{2}, \lambda_{1}} \\
& +\left[B_{l}^{\lambda_{0}} B_{k}^{\lambda_{1}}-B_{k}^{\lambda_{0}} B_{l}^{\lambda_{1}}\right] B_{\lambda_{1}}^{\lambda_{2}} Z_{\lambda_{2}, \lambda_{0}}+B_{k}^{\lambda_{0}} B_{\lambda_{0}}^{\lambda_{1}} B_{\lambda_{1}}^{\lambda_{2}} Z_{\lambda_{2}, l}+B_{l}^{\lambda_{0}} B_{\lambda_{0}}^{\lambda_{1}} B_{\lambda_{1}}^{\lambda_{2}} Z_{\lambda_{2}, k} \\
& +\left[B_{k}^{\lambda_{0}}\left(B_{l}^{\lambda_{1}} B_{\lambda_{0}}^{\mu_{2}}-B_{\lambda_{0}}^{\lambda_{1}} B_{l}^{\mu_{2}}\right)-B_{l}^{\lambda_{0}}\left(B_{k}^{\lambda_{1}} B_{\lambda_{0}}^{\mu_{2}}-B_{\lambda_{0}}^{\lambda_{1}} B_{k}^{\mu_{2}}\right)\right] \underline{Z_{\mu_{2}, \lambda_{1}}} .
\end{aligned}
$$

and canceling the $Z_{\mu_{2}, \lambda_{1}}$ terms and expanding the others by the inductive hypothesis,

$$
\begin{aligned}
= & \left(B_{k}^{\lambda_{0}} B_{l}^{\mu_{1}}-B_{l}^{\lambda_{0}} B_{k}^{\mu_{1}}\right) \underline{Z_{\mu_{1}, \lambda_{0}}}+\left[B_{k}^{\lambda_{0}}\left(B_{l}^{\lambda_{1}} B_{\lambda_{0}}^{\lambda_{2}}-B_{\lambda_{0}}^{\lambda_{1}} B_{l}^{\lambda_{2}}\right)\right. \\
& \left.-B_{l}^{\lambda_{0}}\left(B_{k}^{\lambda_{1}} B_{\lambda_{0}}^{\lambda_{2}}-B_{\lambda_{0}}^{\lambda_{1}} B_{k}^{\lambda_{2}}\right)\right]\left(B_{\lambda_{1}}^{\lambda_{3}} Z_{\lambda_{3}, \lambda_{2}}-B_{\lambda_{2}}^{\lambda_{3}} Z_{\lambda_{3}, \lambda_{1}}+B_{\lambda_{1}}^{\mu_{3}} Z_{\mu_{3}, \lambda_{2}}\right) \\
& +\left[B_{l}^{\lambda_{0}} B_{k}^{\lambda_{1}}-B_{k}^{\lambda_{0}} B_{l}^{\lambda_{1}}\right] B_{\lambda_{1}}^{\lambda_{2}}\left(B_{\lambda_{0}}^{\lambda_{3}} Z_{\lambda_{3}, \lambda_{2}}-B_{\lambda_{2}}^{\lambda_{3}} Z_{\lambda_{3}, \lambda_{0}}+B_{\lambda_{0}}^{\mu_{3}} \underline{Z_{\mu_{3}, \lambda_{2}}}\right) \\
& +B_{k}^{\lambda_{0}} B_{\lambda_{0}}^{\lambda_{1}} B_{\lambda_{1}}^{\lambda_{2}}\left(B_{l}^{\lambda_{3}} Z_{\lambda_{3}, \lambda_{2}}-B_{\lambda_{2}}^{\lambda_{3}} Z_{\lambda_{3}, l}+B_{l}^{\mu_{3}} \underline{Z_{\mu_{3}, \lambda_{2}}}\right)
\end{aligned}
$$




$$
-B_{l}^{\lambda_{0}} B_{\lambda_{0}}^{\lambda_{1}} B_{\lambda_{1}}^{\lambda_{2}}\left(B_{k}^{\lambda_{3}} Z_{\lambda_{3}, \lambda_{2}}-B_{\lambda_{2}}^{\lambda_{3}} Z_{\lambda_{3}, k}+B_{k}^{\mu_{3}} \underline{Z_{\mu_{3}, \lambda_{2}}}\right)
$$

and rearranging,

$$
\begin{aligned}
= & \left(B_{k}^{\lambda_{0}} B_{l}^{\mu_{1}}-B_{l}^{\lambda_{0}} B_{k}^{\mu_{1}}\right) \underline{Z_{\mu_{1}, \lambda_{0}}} \\
& +\left[B_{k}^{\lambda_{0}} B_{l}^{\lambda_{1}}\left(B_{\lambda_{0}}^{\lambda_{2}} B_{\lambda_{1}}^{\lambda_{3}}-B_{\lambda_{1}}^{\lambda_{2}} B_{\lambda_{0}}^{\lambda_{3}}\right)-B_{l}^{\lambda_{0}} B_{k}^{\lambda_{1}}\left(B_{\lambda_{0}}^{\lambda_{2}} B_{\lambda_{1}}^{\lambda_{3}}-B_{\lambda_{1}}^{\lambda_{2}} B_{\lambda_{0}}^{\lambda_{3}}\right)\right. \\
& \left.+B_{l}^{\lambda_{0}} B_{\lambda_{0}}^{\lambda_{1}}\left(B_{k}^{\lambda_{2}} B_{\lambda_{1}}^{\lambda_{3}}-B_{\lambda_{1}}^{\lambda_{2}} B_{k}^{\lambda_{3}}\right)-B_{k}^{\lambda_{0}} B_{\lambda_{0}}^{\lambda_{1}}\left(B_{l}^{\lambda_{2}} B_{\lambda_{1}}^{\lambda_{3}}-B_{\lambda_{1}}^{\lambda_{2}} B_{l}^{\lambda_{3}}\right)\right] Z_{\lambda_{3}, \lambda_{2}} \\
& -\left[B_{k}^{\lambda_{0}}\left(B_{l}^{\lambda_{1}} B_{\lambda_{0}}^{\lambda_{2}}-B_{\lambda_{0}}^{\lambda_{1}} B_{l}^{\lambda_{2}}\right)-B_{l}^{\lambda_{0}}\left(B_{k}^{\lambda_{1}} B_{\lambda_{0}}^{\lambda_{2}}-B_{\lambda_{0}}^{\lambda_{1}} B_{k}^{\lambda_{2}}\right)\right] B_{\lambda_{2}}^{\lambda_{3}} Z_{\lambda_{3}, \lambda_{1}} \\
& -\left[B_{l}^{\lambda_{0}} B_{k}^{\lambda_{1}}-B_{k}^{\lambda_{0}} B_{l}^{\lambda_{1}}\right] B_{\lambda_{1}}^{\lambda_{2}} B_{\lambda_{2}}^{\lambda_{3}} Z_{\lambda_{3}, \lambda_{0}}-B_{k}^{\lambda_{0}} B_{\lambda_{0}}^{\lambda_{1}} B_{\lambda_{1}}^{\lambda_{2}} B_{\lambda_{2}}^{\lambda_{3}} Z_{\lambda_{3}, l}+B_{l}^{\lambda_{0}} B_{\lambda_{0}}^{\lambda_{1}} B_{\lambda_{1}}^{\lambda_{2}} B_{\lambda_{2}}^{\lambda_{3}} Z_{\lambda_{3}, k} \\
& +\left[B_{k}^{\lambda_{0}} B_{l}^{\lambda_{1}}\left(B_{\lambda_{0}}^{\lambda_{2}} B_{\lambda_{1}}^{\mu_{3}}-B_{\lambda_{1}}^{\lambda_{2}} B_{\lambda_{0}}^{\mu_{3}}\right)-B_{l}^{\lambda_{0}} B_{k}^{\lambda_{1}}\left(B_{\lambda_{0}}^{\lambda_{2}} B_{\lambda_{1}}^{\mu_{3}}-B_{\lambda_{1}}^{\lambda_{2}} B_{\lambda_{0}}^{\mu_{3}}\right)\right. \\
& \left.+B_{l}^{\lambda_{0}} B_{\lambda_{0}}^{\lambda_{1}}\left(B_{k}^{\lambda_{2}} B_{\lambda_{1}}^{\mu_{3}}-B_{\lambda_{1}}^{\lambda_{2}} B_{k}^{\mu_{3}}\right)-B_{k}^{\lambda_{0}} B_{\lambda_{0}}^{\lambda_{1}}\left(B_{l}^{\lambda_{2}} B_{\lambda_{1}}^{\mu_{3}}-B_{\lambda_{1}}^{\lambda_{2}} B_{l}^{\mu_{3}}\right)\right] \underline{Z_{\mu_{3}, \lambda_{2}}} .
\end{aligned}
$$

The $Z_{\mu_{3}, \lambda_{2}}$ terms cancel by the inductive hypothesis.

Comparing (3.14) to (3.13), it is apparent that this pattern continues as we expand by the inductive hypothesis; in particular, notice that the upper indices on $Z_{\lambda_{p}, \lambda_{q}}$ or $Z_{\mu_{p}, \lambda_{p-1}}$ always appear as $\lambda_{0}, \lambda_{1}, \ldots, \lambda_{p}$ (or $\mu_{p}$ ), while the lower indices vary through signed permutations of $\left(l, k, \lambda_{0}, \ldots, \lambda_{q}, \ldots, \lambda_{p}\right)$ that end in $\lambda_{p}, \lambda_{q}$. Because these indices satisfy $1 \leq \lambda_{p}<\lambda_{p-1}<\cdots<$ $\lambda_{0}<l$, eventually every $Z_{\lambda_{p}, \lambda_{q}}$ term will reduce by repeated application of (3.9) to terms of the form $Z_{\mu_{p}, \lambda_{p-1}}$. Therefore, by pairing the lower-index permutations by transposition in the third-to-last and fourth-to-last slots, the $Z_{\mu_{p}, \lambda_{p-1}}$ terms always appear as

$$
\cdots\left(B_{i}^{\lambda_{p-1}} B_{j}^{\mu_{p}}-B_{j}^{\lambda_{p-1}} B_{i}^{\mu_{p}}\right) \underline{Z_{\mu_{p}, \lambda_{p-1}}} \quad \text { with } \mu_{p} \geq \lambda_{p-1}, \quad(j, i)<(k, l),
$$

which vanishes by the inductive hypothesis.

Therefore, equation (3.12) reduces by induction to

$$
Z_{l, k}-B_{k}^{\mu_{0}} \underline{Z_{\mu_{0}, l}}=\left(B_{k}^{\lambda_{0}} B_{l}^{\mu_{1}}-B_{l}^{\lambda_{0}} B_{k}^{\mu_{1}}\right) \underline{Z_{\mu_{1}, \lambda_{0}}} .
$$

On $\mathbf{W}_{l}^{+}$, the left-hand side vanishes, so the independence of the $s_{1}+2 s_{2}+\cdots+k s_{k}$ elements $Z_{\mu_{1}, \lambda_{0}}$ required by Cartan's test is equivalent to the condition

$$
\left(B_{l}^{\lambda_{0}} B_{k}^{\mu_{1}}-B_{k}^{\lambda_{0}} B_{l}^{\mu_{1}}\right)=0
$$

projected to $\mathbf{W}_{l}^{+}$for all $\lambda_{0} \leq \mu_{1} \leq k$ and $\lambda_{0}<l$.

This concludes the proof of Theorem 3.2.

Another important subspace is the part of $A$ that is rank-one in $W \otimes U^{*}$; that is, consider

$$
\mathbf{A}^{1}(\varphi)=A \cap\left\{z \otimes \varphi+J \text { for some } z \in W, J \in W \otimes Y^{*}\right\} .
$$

The image of $\mathbf{A}^{1}(\varphi)$ under the projection $\left(W \otimes V^{*}\right) \rightarrow W \otimes U^{*}$ is comprised of rank-one homomorphisms, so the projection $\mathbf{A}^{1}(\varphi) \rightarrow W$ is well-defined, with image

$$
\mathbf{W}^{1}(\varphi)=\left\{z \in W: z \otimes \varphi+J \in A \text { for some } J \in W \otimes Y^{*}\right\} .
$$

The spaces $\mathbf{W}^{-}(\varphi)$ and $\mathbf{W}^{1}(\varphi)$ are distinct, but their relationship is clear:

Lemma 3.3. Suppose that $A$ is an endovolutive tableau. For any $\lambda$,

$$
\mathbf{W}^{1}\left(u^{\lambda}\right)=\left\{z \in \mathbf{W}_{\lambda}^{-}: B_{\mu}^{\lambda} z=\delta_{\mu}^{\lambda} z \forall \mu \leq \ell\right\} .
$$

More generally, for any $\varphi \in U^{*}$,

$$
\mathbf{W}^{1}(\varphi)=\left\{z \in \mathbf{W}^{-}(\varphi):\left(\sum_{\lambda} \varphi_{\lambda} B_{\mu}^{\lambda}-\varphi_{\mu} I\right) z=0 \forall \mu \leq \ell\right\} .
$$


There is nothing to prove; Lemma 3.3 merely states the condition that the $W \otimes U^{*}$ part of $\pi=B(\varphi)(\cdot) z$ is rank-one.

Corollary 3.4. Suppose that $A$ is an endovolutive tableau. For almost all $\varphi, \operatorname{dim} \mathbf{W}^{1}(\varphi)=s_{\ell}$.

Proof. For almost all $\varphi \in U^{*}$, we have $\min \left\{i: \varphi_{i} \neq 0\right\}=1$ and $\max \left\{i: \varphi_{i} \neq 0\right\}=\ell$. So, $\mathbf{W}^{-}(\varphi)=\mathbf{W}_{1}^{-}$has dimension $s_{1}$. For each $\mu=1, \ldots, \ell$ the condition $\left(\varphi_{\lambda} B_{\mu}^{\lambda}-\varphi_{\mu} I\right) z=0$ has no $\mathbf{W}_{\mu}^{-}$component, but the $\mathbf{W}_{\mu}^{+}$component has rank up to $s_{\mu-1}-s_{\mu}$. The rank falls if and only if $\varphi_{\mu}$ is an eigenvalue of $\sum_{\lambda<\mu} \varphi_{\lambda} B_{\mu}^{\lambda}$. By fixing $\varphi_{1}, \varphi_{2}, \ldots, \varphi_{\mu-1}$ and varying $\varphi_{\mu}$, we can see that this condition achieves its maximum rank for a Zariski-open set of values of $\varphi$. Then $\operatorname{dim} \mathbf{W}^{1}(\varphi)=s_{1}-\left(s_{1}-s_{2}\right)-\cdots-\left(s_{\ell-1}-s_{\ell}\right)=s_{\ell}$.

Note that, unlike with $\mathbf{W}^{-}(\varphi)$, the definition of $\mathbf{W}^{1}(\varphi)$ does not rely on the basis; it requires only a splitting of $0 \rightarrow U^{*} \rightarrow V^{*} \rightarrow Y \rightarrow 0$ to decide where $J$ takes values. For this reason, it is the space studied in the homological references. The space $\mathbf{W}^{1}(\varphi)$ is the subject of Theorem 3.5, which is known colloquially as "Guillemin normal form". It is called ${ }^{3}$ Lemma $^{4.1}$ in [3] and Proposition 6.3 in Chapter VIII of [1]. Theorem 3.5 is crucial to the study of partial differential equations and exterior differential systems because it reveals the intimate relationship between involutivity, overdetermined Cauchy initial-value problems, and the characteristic variety [4].

Theorem 3.5 (Guillemin). Suppose that $A$ is involutive. For every $\varphi \in U^{*}$ and $v \in V$, the restricted homomorphism $\left.B(\varphi)(v)\right|_{\mathbf{W}^{1}(\varphi)}$ is an endomorphism of $\mathbf{W}^{1}(\varphi)$. Moreover, for all $v, \tilde{v} \in V$,

$$
\left.[B(\varphi)(v), B(\varphi)(\tilde{v})]\right|_{\mathbf{W}^{1}(\varphi)}=0 .
$$

Guillemin's original proof of Theorem 3.5 relies on several subtle homological results. Each of those results can be reproven using Theorem 3.2 and elementary linear algebra. For example, here is the key result:

Corollary 3.6 (Quillen, Guillemin). If $A$ is involutive, then $\left.A\right|_{U}$ is involutive, and the natural map between prolongations $A^{(1)} \rightarrow\left(\left.A\right|_{U}\right)^{(1)}$ is bijective.

Proof. This is Theorem A in [3], where it is proven with a large diagram chase using Quillen's exactness theorem from [7]. But, using Theorem 3.2, this is immediate, as the quadratic condition still holds if the range of indices $\lambda, \mu, i, j$ is truncated at $\ell$ (or greater). In particular, the generators $\left(z_{\mu}^{a}\right)_{a \leq s_{\mu}}$ of $A$ are preserved. As explored in the proof of Theorem 3.2, the contact relation $z_{\mu}^{a}=Z_{\mu, i}^{a} u^{i}$ gives coordinates $Z_{\mu, i}^{a}$ to the prolongation $A^{(1)} \subset A \otimes V^{*}$, and the $s_{1}+2 s_{2}+\cdots+\ell s_{\ell}$ independent generators are $\underline{Z_{\mu, \lambda}^{a}}$ with $a \leq s_{\mu}$ and $\lambda \leq \mu$. These generators remain independent under restriction to $U$, too.

Theorems 3.2 and 3.5 appear to be very similar in the sense that each implies linear and quadratic conditions on the symbol coefficients of involutive tableaux. However, Theorem 3.2 is strictly stronger, as it provides equivalence, whereas Theorem 3.5 is a unidirectional implication. For example, consider this tableau with characters $(3,1,0)$ :

$$
\pi=\left[\begin{array}{ccc}
\pi_{1}^{1} & \pi_{2}^{1} & P_{1} \pi_{1}^{1}+P_{2} \pi_{1}^{2}+P_{3} \pi_{1}^{3}+Q \pi_{2}^{1} \\
\pi_{1}^{2} & \pi_{1}^{3} & T_{2} \pi_{1}^{2}+T_{3} \pi_{1}^{3} \\
\pi_{1}^{3} & 0 & R_{3} \pi_{1}^{3}
\end{array}\right]
$$

\footnotetext{
${ }^{3}$ In these references, the domain is restricted to $v \in Y$, but that limitation is artificial.
} 
with symbol maps arranged as

$$
\begin{aligned}
& \left(\begin{array}{lll}
1 & 0 & 0 \\
0 & 1 & 0 \\
0 & 0 & 1
\end{array}\right), \quad\left(\begin{array}{ccc}
0 & 0 & 0 \\
0 & 0 & 1 \\
0 & 0 & 0
\end{array}\right), \quad\left(\begin{array}{ccc}
P_{1} & P_{2} & P_{3} \\
0 & T_{2} & T_{3} \\
0 & 0 & R_{3}
\end{array}\right), \\
& \left(\begin{array}{ll}
1 & \\
&
\end{array}\right), \quad\left(\begin{array}{l}
Q \\
\end{array}\right) \text {. }
\end{aligned}
$$

This tableau is written in generic bases, and it is endovolutive. It has $\mathbf{W}^{-}\left(u^{1}\right)=\left\langle w_{1}, w_{2}, w_{3}\right\rangle$, $\mathbf{W}^{-}\left(u^{1}\right)=\left\langle w_{1}, w_{2}\right\rangle$, and $\mathbf{W}^{1}\left(u^{1}\right)=\left\langle w_{1}, w_{2}\right\rangle$. For $\varphi=\varphi_{1} u^{1}+\varphi_{2} u^{2} \in U^{*}$ with $\varphi_{2} \neq 0$, it has $\mathbf{W}^{1}(\varphi)=\left\langle w_{1}\right\rangle$. Moreover, for all $\varphi=\varphi_{1} u^{1}+\varphi_{2} u^{2} \in U^{*}$ and all $v \in V$, the restricted map $\left.B(\varphi)(v)\right|_{\mathbf{W}^{1}(\varphi)}$ is an endomorphism of $\mathbf{W}^{1}(\varphi)$, and the commutativity condition (3.15) holds. However, by Theorem 3.2, this tableau is involutive if and only if $T_{2}=R_{3}$.

Theorem 3.5 sets conditions on operators acting in dimension $s_{\ell}$, whereas Theorem 3.2 sets conditions on operators acting in dimension $s_{1}$. These additional conditions allow Theorem 3.2 to describe the variety of involutive tableaux, a strict subvariety of the endovolutive tableaux satisfying (3.15).

\section{Discussion}

Theorem 3.2 is the first step to answering a very fundamental open question, which is expressed in footnote 7 in Chapter IV of [1]: "What is the dimension of the space of involutive tableaux with certain fixed Cartan characters?" Lemma 3.1 and Theorem 3.2 together give an algebraic ideal whose variety contains every involutive tableaux, each expressed in a preferred basis. It is not immediately clear how to account for non-Borel basis changes or in what sense the endovolutive expression of $B_{i}^{\lambda}$ is unique for a given abstract tableau, but the recursive nature of the proof suggests that Theorem 3.2 provides minimal set of generators for this ideal.

Because it is easy to program into computer algebra systems ${ }^{4}$, Theorem 3.2 allows us to explore the moduli of involutive tableaux and search for interesting new families of involutive partial differential equations with peculiar geometric properties. More generally, I hope it lowers the barrier for future researchers of exterior differential systems and Lie pseudogroups who want to understand and apply the profound results of [7] and [3].

\section{Acknowledgments}

Thanks to Deane Yang for several helpful conversations. Thanks also to the anonymous referees, whose suggestions improved the style and focus of this article significantly.

\section{References}

[1] Bryant R.L., Chern S.S., Gardner R.B., Goldschmidt H.L., Griffiths P.A., Exterior differential systems, Mathematical Sciences Research Institute Publications, Vol. 18, Springer-Verlag, New York, 1991, available at http://library.msri.org/books/Book18/MSRI-v18-Bryant-Chern-et-al.pdf.

[2] Carlson J., Green M., Griffiths P., Variations of Hodge structure considered as an exterior differential system: old and new results, SIGMA 5 (2009), 087, 40 pages, arXiv:0909.2201.

[3] Guillemin V., Some algebraic results concerning the characteristics of overdetermined partial differential equations, Amer. J. Math. 90 (1968), 270-284.

\footnotetext{
${ }^{4}$ I am writing an open-source package called Symbol for Sage using this approach. You can help at https: //bitbucket.org/curieux/symbol_sage.
} 
[4] Guillemin V.W., Quillen D., Sternberg S., The integrability of characteristics, Comm. Pure Appl. Math. 23 (1970), 39-77.

[5] Kruglikov B., Lychagin V., Spencer $\delta$-cohomology, restrictions, characteristics and involutive symbolic PDEs, Acta Appl. Math. 95 (2007), 31-50, math.DG/0503124.

[6] Malgrange B., Cartan involutiveness = Mumford regularity, in Commutative Algebra (Grenoble/Lyon, 2001), Contemp. Math., Vol. 331, Amer. Math. Soc., Providence, RI, 2003, 193-205.

[7] Quillen D.G., Formal properties of over-determined systems of partial differential equations, Ph.D. Thesis, Harvard University, 1964.

[8] Smith A.D., Degeneracy of the characteristic variety, arXiv:1410.6947.

[9] Yang D., Involutive hyperbolic differential systems, Mem. Amer. Math. Soc. 68 (1987), no. 370, xii+93 pages. 